\title{
Intraoral Local Anesthesia and Ocular Complications
}

\author{
Kavitha Patil, Karishma Munoli, Vinod Kumar, Kartik Venkataraghavan
}

\begin{abstract}
Background: Ocular complications due to intraoral local anesthesia are rare but most distressing to the dentist and patient. Ocular complications after local anesthetic injections are rarely reported in the literature and these complications include strabismus, ptosis, diplopia, blindness, ophthalmoplegia, loss of accommodation.

The dentist must be vigilant and beware of ocular complications when performing local anesthetic nerve blocks.
\end{abstract}

Aim: The aim and objective of this review is to help the dental surgeons to understand about the causes, mechanisms and symptoms of ocular complications to enable them to diagnose, manage and prevent those complications.

Materials and methods: The revision included a systematic literature search of past 10 years with keywords dental anesthesia, ocular complications, blindness after dental anesthesia, diplopia following dental anesthesia.

Papers for review were chosen from the selected articles. When data did not appear sufficient or were inconclusive; recommendations were based upon expert or consensus opinion by experienced researchers and clinicians.

Conclusion: The various ocular complications, its causes, mechanisms and management are emphasized in this review paper. As per literature review ocular complications are rare to occur but they are most distressing, clinicians should be aware of complications and should be able to diagnose and understand the complications for timely prevention and effective management.

Keywords: Diplopia, Ocular complications, Dental anesthesia.

How to cite this article: Patil K, Munoli K, Kumar V, Venkataraghavan K. Intraoral Local Anesthesia and Ocular Complications. World J Dent 2013;4(2):108-112.

Source of support: Nil

Conflict of interest: None declared

\section{INTRODUCTION}

Intraoperative pain control by means of local anesthesia is an intrinsic part of clinical practice in dentistry. ${ }^{1}$ There are local and systemic complications following local anesthetic injections in dentistry. Although there may be some rare systemic complications; most complications are local and temporary. Local complications include hematoma formation, pain at the sight of injection due to nerve impingement or injection into a muscle, trismus and facial nerve paralysis, etc. ${ }^{2}$ Ocular disturbances have known to occur as rare complications due to intraoral local anesthesia. ${ }^{3}$ Despite being described as a local type of complications due to intraoral local anesthesia, the ocular alterations are quite uncommon, representing approximately $0.1 \%$ of the complications. ${ }^{4}$

There are few studies which report various ocular complications. A comprehensive review of literature provides an overview of ocular complications due to local anesthetic injection techniques in dentistry.

\section{COMMON OCULAR COMPLICATIONS}

Commonly occurring ocular complications due to local anesthetic injections in dentistry are as follows: ${ }^{5}$

1. Diplopia

2. Strabismus

3. Ptosis

4. Loss of accommodation

5. Ophthalmoplegia

6. Amaurosis (blindness).

These complications following dental anesthesia are usually temporary and wear off along with the effects of local anesthesia. Very rarely does permanent blindness occur and is usually accompanied by other systemic presentations.

Various causes for ocular complications due to intraoral local anesthesia are as follows: $:^{6,7}$

- Diffusion

- Inadvertent needle penetration into the orbit

- Venous injection

- Retrograde arterial injection

- Sympathetic impulse generation

- Embolism.

The nerve blocks which are prone for ocular complications are as follows:

1. Posterior superior alveolar nerve block

2. Inferior alveolar nerve block.

For the past 45 years, ocular complications after middle or posterior superior alveolar nerve block were reported twice more frequent than inferior alveolar nerve block.

The posterior superior alveolar nerve is a branch of the maxillary division of the trigeminal nerve (cranial nerve $\mathrm{V}$ ) arising in the pterygopalatine fossa just before it enters the infraorbital canal. It gives out two branches, one providing sensory innervations to the mucous membrane of the maxillary sinus and the second to the alveoli, periodontal ligaments and pulpal tissues of maxillary molars.

The posterior superior nerve block is mainly given to achieve anesthesia of maxillary molars (except mesiobuccal root of first molar) and surrounding structures. 
The inferior alveolar nerve is the largest branch of the mandibular division of the trigeminal nerve. It travels down the lateral pterygoid muscle and lateroposterior to the lingual nerve and travels up to the mental foramen where it gives its terminal branches: incisive nerve and mental nerve.

During its course, the inferior alveolar nerve is always accompanied by the inferior alveolar artery (a branch of the internal maxillary artery) and the inferior alveolar vein. The artery lies just anterior to the nerve.

The inferior alveolar nerve block is mainly given to achieve anesthesia of mandibular teeth and surrounding structures.

\section{SYMPTOMS}

The symptoms experienced are:

1. Blurring of vision

2. Double vision

3. Decreased sensation on the lateral aspect of the upper and lower eyelids

4. Squinting

5. Loss of vision

6. Dropping of the upper eyelid

7. Difficulty in reading due to paralysis of accommodation

8. Transient dizziness.

\section{PATHOGENIC MECHANISM OF COMPLICATIONS}

Various mechanisms for ocular complications due to dental anesthesia are as follows:

- Diplopia: The possible mechanism of the ocular complications is intravascular injection of the local anesthetic solution. Since the inferior alveolar artery lies in close proximity to the inferior alveolar nerve, a chance of an intravascular injection is quite high. Due to pressure with which the anesthetic solution is injected, the solution is forced back to the maxillary artery. The middle meningeal artery may arise from the maxillary artery; also the origins of middle meningeal artery and the inferior alveolar artery are closely situated, which leads to the entry of the anesthetic solution. The ophthalmic branch of the middle meningeal artery may anastomose with the lacrimal artery. The blood supply to the lateral rectus muscle is derived from the lacrimal artery and the lateral muscular trunk of the ophthalmic artery. Hence, the intravascular injection of the local anesthetic may lead to the paralysis of the lateral rectus causing diplopia. ${ }^{8,9}$

Diplopia may also be caused by inadvertent entry of the anesthetic solution into the venous system, which drains into the pterygoid plexus and further the cavernous sinus. The abducent nerve being more susceptible as it passes through the cavernous sinus leading to paralysis of the lateral rectus muscle causing diplopia. ${ }^{10}$

- Paralysis of the eye: Inadvertent entry of local anesthetic solution into venous system which drains into pterygoid plexus and later into cavernous sinus. The retrograde flow of local anesthetic solution into cavernous cause's pressure in the sinus resulting into paralysis of oculomotor nerve which is in close proximity to it. ${ }^{11,12}$

- Blindness (amaurosis): The central retinal artery is a small branch of the ophthalmic artery; any anesthetic solution flowing through the middle meningeal artery may enter the ophthalmic artery and further the retinal artery causing blindness and loss of pupillary light reflex.

A reflex vasospasm of the central retinal artery results in ischemia and necrosis of retinal tissue causing permanent blindness. ${ }^{13}$

So far in literature there are very few reported cases of permanent blindness due to intraoral local anesthesia. De Keyzer et $\mathrm{al}^{14}$ and Rishiraj et $\mathrm{al}^{15}$ reported permanent blindness in one eye following administration of intraoral local anesthesia. Walsh ${ }^{16}$ described a case of permanent amaurosis after dental anesthesia of upper teeth; he attributed this to the oily nature of local anesthetic solution containing procaine hydrochloride which resulted in the embolism of retinal artery.

- Ptosis, strabismus and loss of accommodation: The power of accommodation is dependent on the elasticity of the lens capsule and the contraction of the ciliary muscles supplied by the short ciliary nerves. The short ciliary nerves are the postganglionic fibers of the ciliary ganglion, which are provided parasympathetic preganglionic fibers by the oculomotor nerve (cranial nerve III). When there is paralysis of oculomotor nerve due to injury or anesthesia it results into ptosis, strabismus, dilatation of the pupil and loss of power of accommodation as the sphincter pupillae, the ciliary muscle and the internal rectus are paralyzed. Occasionally paralysis may affect only a part of the nerve. Thus there may be internal strabismus from spasm of the internal rectus, accommodation for near objects only from spasm of the ciliary muscle or miosis (contraction of the pupil) from irritation of the sphincter of the pupil. ${ }^{12}$

- Some literatures describe Horner like syndrome with ptosis, miosis, vascular dilatation of the conjunctiva and generalized rash over the face, neck shoulder and arm. The mechanism suggested is the accidental injection of local anesthetic drug into the stellate ganglion. ${ }^{17}$ 


\section{Literature Review}

Penarrocha-Diago and Sanchis-Bielsa (2000) presented a series of 14 cases of ophthalmologic complications after intraoral anesthesia of the posterior superior alveolar nerve. The most commonly encountered symptoms were diplopia, mydriasis, palpebral ptosis, and abduction difficulties of the affected eye. In all cases, these effects occurred a few minutes after injection of the anesthetic, followed by complete resolution without sequelae on cessation of the anesthetic effect. The pathogenic mechanism underlying such ophthalmologic disorders is discussed in terms of a possible diffusion of the anesthetic solution toward the orbital region. ${ }^{18}$

Joseph Horowitz et al (2005) presented three case reports; two patients had ipsilateral optic neuropathy and one patient had an ipsilateral abduction deficit and a dilated, poorly reactive pupil immediately after anesthesia of upper alveolar teeth. In all but three cases, the deficits were temporary. Diffusion, inadvertent needle penetration into the orbit and intravascular injection was postulated as the mechanism by which the anesthetic agent reaches the cavernous sinus or orbit to cause the deficits. ${ }^{6}$

Magliocca et al (2006) presented a case of 36-year-old female patient who developed diplopia and an ipsilateral lateral rectus paresis following local anesthetic administration to remove a left maxillary second molar. Complete resolution occurred within 3 hours. The clinical examination and management plan were reviewed for this uncommon occurrence. The relevant anatomical pathways are discussed and illustrated with photographs. ${ }^{19}$

In 2006 Wei Cheong Ngeow, Chen Kiong Shim and Wen Lin Chai presented a case report of two patients who developed transient loss of power of accommodation of the eye resulting in blurred vision after routine inferior alveolar nerve blocks on the ipsilateral side. Clear vision returned within 10 to 15 minutes after completion of the blocks. They explained that possible mechanism for this phenomenon is accidental injection into the neurovascular bundle of local anesthetic agents, which were carried via the blood to the orbital region. This resulted in paralysis of a branch of cranial nerve III, the short ciliary nerves that innervate the ciliary muscle, which controls accommodation. ${ }^{20}$

Williams and Williams (2011) reported the unusual case of a young patient who experienced amaurosis, total ophthalmoplegia, complete upper eyelid ptosis, mydriasis and periorbital blanching following inferior alveolar nerve anesthesia. ${ }^{21}$

Kini, Kharkar and Kini (2011) have explained details about an iatrogenic paresis of the abducent nerve and partial palsy of the oculomotor nerve leading to diplopia, strabismus and ptosis following a posterior superior alveolar nerve block and extraction of maxillary right second molar. The patient was treated symptomatically, and the recovery was uneventful. ${ }^{22}$

Jose-Maria Aguada et al (2011) carried out a review of 19 articles to find out the incidence and type of ocular complications after intraoral local anesthesia. They found that ophthalmological complications are seldom a problem, diplopia being the most common complication (65\%), which coincides with the data from other authors. Almost all of the complications were of a temporary nature, with an average recovery time of 68 minutes. The authors appear to indicate that an intravascular injection of the anesthetic was the cause of the problem, and therefore, it should be avoided in order to prevent accidents at the ocular level. ${ }^{4}$

Steenen et al (2012) reported a case in which a patient with right lateral rectus muscle palsy and blurred vision after bimaxillary anesthesia. Signs, symptoms and pathophysiologic hypotheses of these complications are presented. $^{23}$

Yoon and Chussid (2012) presented a case report of 7-year-old male patient who developed palpebral ptosis and paralysis of the extraocular muscles following local anesthetic administration for a major operative procedure on the permanent mandibular left first molar. Complete resolution occurred within approximately 30 minutes. ${ }^{24}$

Verma, Rajan and Prabhu (2012) have reported two rare cases of transient isolated amaurosis. Both the instances of amaurosis were seen after inferior alveolar nerve blocks. The condition was transient and resolved without any active intervention. The etiology in the present case was believed to be intravascular injection. ${ }^{7}$

Steenen et al (2012) reviewed the literature from the years 1936 to 2011 and showed 131 cases with this type of complication and also presented a case report of a patient with right lateral rectus muscle palsy and blurred vision after bimaxillary anesthesia. Signs, symptoms and pathophysiologic hypotheses of these complications were presented. $^{23}$

\section{Prevention and Management of Complications}

Prevention is better than cure. Hence, it is always better to avoid anesthetic misadventure. Most of the complications occurring are temporary and recovery is almost immediate. In most cases, patients complaining of diplopia, blurring of vision or loss of accommodation, gain back vision within 5 to 60 minutes after the onset. In certain cases it may exceed to up to 4 to 5 hours and rarely up to 24 hours. 
Permanent blindness, a very rare entity may occur due to the ischemia and necrosis of the retinal tissue caused by the reflex vasospasm of the retinal artery when a substantial amount of anesthetic agent enters the retinal artery through the ophthalmic artery.

1. Reassurance of the patient is the most important step.

2. An eye patch may be applied to the affected eye, to protect the cornea, due to the anesthetic effects as well as to restore functional monocular vision.

3. An evaluation by the ophthalmologist is quintessential in case the complications last for longer than 5 hours.

4. When visual acuity is affected patients should be warned against difficult tasks like driving, using sharp objects and going home unaccompanied until the anesthetic effect wears off.

5. Care must be taken especially when procedures are attempted on children, who cannot express the symptoms. Therefore, the clinicians must be vigilant during such times, and must foresee ocular complications.

The precautions one needs to take to prevent ophthalmic complications are:

1. Avoid injections into vascular systems

2. Aspirate prior to injection and slowly administer the anesthetic agent

3. Knowing the anatomical structures properly with regard to the particular nerve block

4. Follow the accepted injection techniques and procedures.

\section{CONCLUSION}

Ocular complications of local anesthetic injections in dentistry although rare but it should not be undermined.

Most of ocular complications are temporary; they can revert back to normalcy after anesthetic affect wears off. Dental surgeon should be well aware of the ocular complications and its prevention and immediate management. A referral to ophthalmologist is recommended if there are lingering ocular disturbances after anesthesia wears off.

Prompt diagnosis and appropriate management prevents permanent ocular damage.

\section{REFERENCES}

1. Haas DA, Lennon D. Local anaesthesia use by dentists in Ontario. J Can Dent Assoc 1995;61(4):297-304.

2. Pogrel MA, Thamby S. Permanent nerve involvement resulting from inferior alveolar nerve blocks. J Am Dent Assoc 2000;131(7):901-07.
3. Blanton LP, Jeske HA. Avoiding complications in local anesthesia induction-anatomical considerations. J Am Dent Assoc 2003;134:888-93.

4. Aguado-Gil JM, Barona-Dorado C, Lillo-Rodríguez JC, De la Fuente-González DS, Martínez-González JM. Occular complications following dental local anesthesia. Med Oral Patol Oral Cir Bucal 2011 Aug1;16(5):e688-93.

5. Wilkie GJ. Temporary uniocular blindness and ophthalmoplegia associated with a mandibular block injection. A case report. Aust Dent J 2000;45(2):131-33.

6. Horowitz J, Almong Y, Wolf A, Buckman G, Geyer O. Ophthalmic complications of dental anesthesia: Three new cases. J Neuroopthalmol 2005;25(2):95-100.

7. Verma DK, Rajan R, Prabhu S. Ipsilateral, isolated amaurosis after inferior alveolar nerve block: Report of two cases. Oral Maxillofac Surg 2013 Mar;17(1):73-75

8. Goldenberg AS. Transient diplopia as a result of block injections. Mandibular and posterior superior alveolar. NY State Dent J 1997;635(5):29-31.

9. Fish LR, Mcintire DN, Johnson L. Temporary paralysis of cranial nerves III, IV and VI after a Gow-Gates injection. J Am Dent Assoc 1989;119(1):127-28.

10. Walker M, Drangsholt M, Czartoski TJ, Longstreth WT. Dental diplopia with transient abducens palsy. Neurology 2004;63:2449-50.

11. Lee CK. Ocular complications after inferior alveolar nerve block. Hong Kong Medical Diary 2006 Aug;11(8):4-5.

12. Pickering T, Howden R. Gray's anatomy, descriptive and surgical (15th ed). London: Chancellor Press 1985:703,805-30.

13. Cole JK. Ocular complications resulting from intra-arterial injection during inferior alveolar nerve anesthesia. Anesth Prog 1982;29(1):9-10.

14. De Keyer K, Tassignon MJ. Case report: Acute unilateral loss of visual acuity after a visit to the dentist: An unusual complication after the use of an anesthetic combined with adrenaline. Rev Belge Med Dent 2004;59:30-33.

15. Rishiraj B, Epstein JB, Fine D, Nabi S, Wade NK. Permanent vision loss in one eye following administration of local anesthesia for a dental extraction. Int J Oral Maxilofac Surg 2005;34(2):220-23.

16. Walsh FB. Clinical neuro-ophthalmology (2nd ed).Baltimore: Williams and Wilkins 1957:1202.

17. Campbell RI, Mercuri LG, Van Sickels J. Cervical sympathetic block following intraoral local anesthesia. Oral Surg Oral Med Oral Pathol 1979; 47(3):223-26.

18. Penarrocha-Diago M, Sanchis-Bielsa JM. Ophthalmologic complications after intraoral local anaesthesia with articaine. Oral Surg Oral Med Oral Pathol Oral Radiol Endod 2000 Jul;90(1):21-24.

19. Magliocca KR, Kessel NC, Cortright GW. Transient diplopia following maxillary local anaesthetic injection. Oral Surg Oral Med Oral Path Oral Radiol Endod 2006 Jun;101(6):730-33.

20. Ngeow WC, Shim CK, Chai WL. Transient loss of power of accommodation in 1 eye following inferior alveolar nerve block: Report of 2 cases. J Can Dent Assoc 2006 Dec;72(10):927-31.

21. Williams JV, Williams LR, Colbert SD, Revington PJ. Amaurosis, ophthalmoplegia, ptosis, mydriasis and periorbital blanching following inferior alveolar nerve anaesthesia. Oral Maxillofac Surg 2011 Mar;15(1):67-70.

22. Kini YK, Kharkar VR, Kini AY. Transient diplopia with ipsilateral abducent nerve palsy and ptosis following a maxillary 
local anesthetic injection: A case report and review of literature. Oral Maxillofac Surg 2012 Dec;16(4):373-75.

23. Steenen SA, Dubois L, Saeed P, de Lange J. Ophthalmologic complications after intraoral local anesthesia: Case report and review of literature. Oral Surg Oral Med Oral Path Oral Radiol Endod 2012 Jun;113(6):e1-5.

24. Yoon RK, Chussid S. Ocular complications following an inferior alveolar nerve block on a child patient: A review of the literature and report of a case. Pediatr Dent 2012;34(4):343-46.

\section{ABOUT THE AUTHORS}

\section{Kavitha Patil}

Professor and Head, Department of Ophthalmology, Raichur Institute of Medical Sciences, Raichur, Karnataka, India

\section{Karishma Munoli}

Senior Resident, Department of Ophthalmology, Raichur Institute of Medical Sciences, Raichur, Karnataka, India

\section{Vinod Kumar (Corresponding Author)}

Reader, Department of Pediatric and Preventive Dentistry, AME'S Dental College and Hospital, Raichur-584101, Karnataka, India Phone: +91-9845971171, e-mail: dr.vinod990@gmail.com

\section{Kartik Venkataraghavan}

Professor and Head, Department of Pedodontics and Preventive Dentistry, College of Dental Sciences and Research Centre Ahmedabad, Gujarat, India 